\title{
Biliopleural fistula following percutaneous transhepatic cholangiodrainage and irradiation biliary stent insertion successfully treated with coil embolization: a case description
}

\author{
Fulei Gao ${ }^{1,2 \#}$, Tianfan Pan $^{1 \#}$, Yong Wang ${ }^{2}$, Guangyu Zhu ${ }^{2}$, Yadong Feng ${ }^{3}$, Cuifang Zhu ${ }^{1}$, Jinhe Guo ${ }^{2}$ \\ ${ }^{1}$ Department of Interventional Radiology, Jiangyin People's Hospital, Jiangyin, China; ${ }^{2}$ Center of Interventional Radiology and Vascular Surgery, \\ Zhongda Hospital, Southeast University, Nanjing, China; ${ }^{3}$ Department of Gastroenterology, Zhongda Hospital, Southeast University, Nanjing, \\ China
}

\#These authors contributed equally to this work.

Correspondence to: Jinhe Guo. Center of Interventional Radiology and Vascular Surgery, Zhongda Hospital, Medical School of Southeast University, 87 Ding Jia Qiao Road, Nanjing 210009, China. Email: jinheguo@sina.com.

Submitted Apr 26, 2021. Accepted for publication Sep 22, 2021.

doi: 10.21037/qims-21-447

View this article at: https://dx.doi.org/10.21037/qims-21-447

\section{Introduction}

Biliopleural fistula (BF) following percutaneous transhepatic cholangiodrainage (PTCD) and irradiation biliary stent insertion is an exceedingly rare clinical entity. The present study describes the case of a 62 -year-old man with malignant biliary obstruction complicated by $\mathrm{BF}$ after PTCD and irradiation biliary stent insertion for this rare disease. After successful closure of the fistula with transcatheter coil embolization, the patient was discharged without any complications or symptoms.

\section{Case presentation}

All procedures performed in studies involving human participants were in accordance with the ethical standards of the institutional and/or national research committee(s) and with the Helsinki Declaration (as revised in 2013). Written informed consent was obtained from the patient for publication of this case report and accompanying images. A copy of the written consent is available for review by the editorial office of this journal. A 62-yearold man was admitted to our department on October 10, 2020 due to icteric skin and sclera accompanied with dark urine for 1 week. He underwent distal pancreatectomy and splenectomy in July 2016. Pathology revealed a (pancreatic body and tail) ductal adenocarcinoma. Magnetic resonance cholangiopancreatography (MRCP) detected compression of the upper segment of the common bile duct (CBD) caused by retroperitoneal lymph node metastasis and dilatation of the intrahepatic bile duct (IHD) and CBD (Figure 1). Laboratory examination revealed the total bilirubin (TBIL) and direct bilirubin (DBIL) levels to be $88.8 \mu \mathrm{mol} / \mathrm{L}$ (normal range, $2.0-20.0 \mu \mathrm{mol} / \mathrm{L}$ ) and $66.7 \mu \mathrm{mol} / \mathrm{L}$ (normal range, 0-6.0 $\mu \mathrm{mol} / \mathrm{L}$ ), respectively. Also, the patient's Carbohydrate antigen 199 (CA199) and carcinoembryonic antigen (CEA) levels increased to $518 \mathrm{U} / \mathrm{mL}$ (normal range, 0-34.0 U/mL) and $20.5 \mathrm{ng} / \mathrm{mL}$ (normal range, $0-4.7 \mathrm{ng} / \mathrm{mL}$ ), respectively.

According to the results, digital subtraction angiography (DSA)-guided PTCD and irradiation biliary stent insertion were successively performed to eliminate jaundice on October 13, 2020. The irradiation stent (Nanjing MicroInvasive Medical Inc., Nanjing, China) loaded with 16 radioactive ${ }^{125} \mathrm{I}$ seeds (type 6711 , particle activity $0.6 \mathrm{mCi}$, half-life 59.4 days, energy $27-35 \mathrm{keV}$; Chinese Atomic Energy Science Institution, Beijing, China) was placed into the diseased bile duct. After deployment of the irradiation stent, the self-expanding biliary stent $(6 \mathrm{~mm} \times$ 80 mm, Cordis, Johnson \& Johnson Inc., New Brunswick, NJ, USA) was immediately implanted to overlap the irradiation stent. An 8.5 FR pig-tailed drainage tube (Cook Medical Inc., Bloomington, IN, USA) was inserted 
into the CBD after stent placement (Figure $2 A, 2 B$ ). The coronal view of the abdominal CT detected the catheter via the right liver through the right costophrenic angle without right pleural effusion (Figure 3). Liver function re-examination after 1 week showed that the TBIL and DBIL levels were 23.6 and $19.2 \mu \mathrm{mol} / \mathrm{L}$, respectively. Physical examination revealed an improvement in the jaundice symptoms. Arterial infusion chemotherapy was performed on October 26, 2020. The following regimen was administered via the gastroduodenal artery: $1,200 \mathrm{mg}$

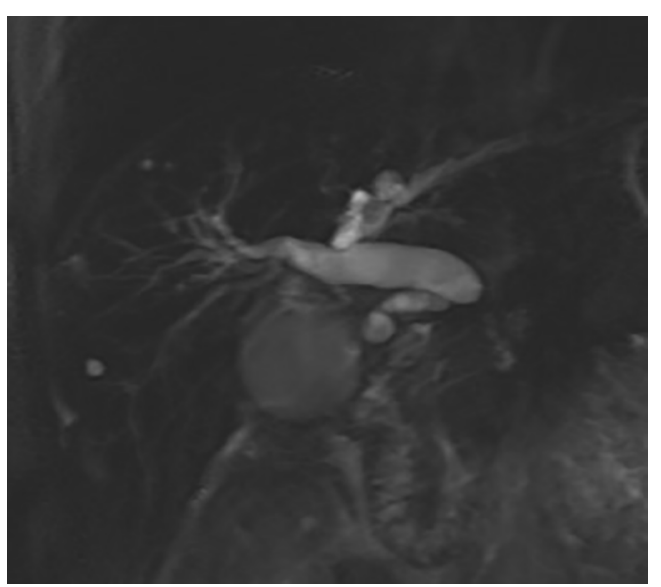

Figure 1 Magnetic resonance cholangiopancreatography (MRCP) detected compression of the upper segment of common bile duct (CBD) caused by retroperitoneal lymph node metastasis and dilatation of the (IHD) and CBD. of gemcitabine from hour 0 to 1 and $200 \mathrm{mg}$ of Abraxane from hour 1 to 2 . Cholangiography performed 15 days later showed the patency of the irradiation biliary stent, and the drainage tube was subsequently removed.

On October 30, 2020, the patient suddenly developed symptoms of chest suffocation and shortness of breath. Physical examination showed disappearance of respiratory sounds on auscultation in the right lung and tenderness in the right upper abdomen. The coronal view of the abdominal CT showed right pleural effusion and perihepatic effusion (Figure 4). A rapid ultrasound-guided thoracentesis was performed, and greenish pyogenic fluid was collected.

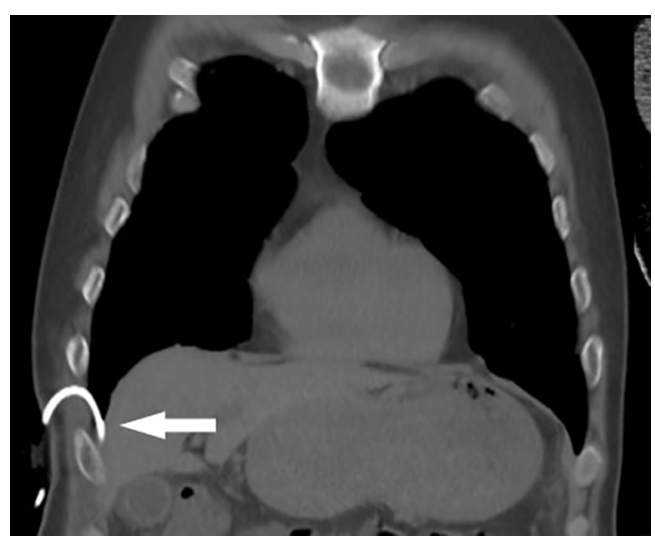

Figure 3 The coronal view on abdominal computed tomography (CT) detected the catheter via the right liver through the right costophrenic angle (white arrow).
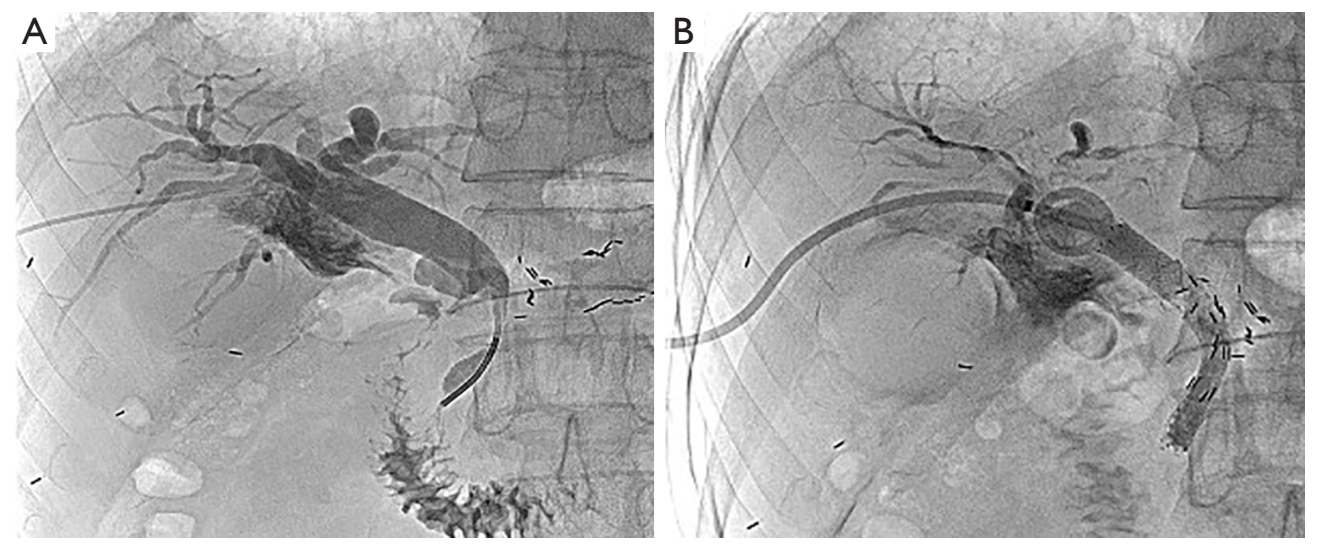

Figure 2 Digital subtraction angiography (DSA)-guided percutaneous transhepatic cholangiodrainage (PTCD) and irradiation biliary stent insertion were successively performed to eliminate jaundice. (A) Cholangiography showed the compression of the upper segment of common bile duct (CBD) and dilatation of the intrahepatic bile duct (IHD) and CBD, as shown in magnetic resonance cholangiopancreatography (MRCP). (B) The irradiation stent loaded with 16 radioactive ${ }^{125} \mathrm{I}$ seeds and a self-expanding biliary stent were placed into the diseased bile duct. An $8.5 \mathrm{FR}$ pig-tailed drainage tube was inserted into the CBD after stent insertion. 
We installed a drainage tube into the right pleural space for continuous drainage. Biochemical analysis of the fluid revealed bile with neutrophilic leukocyte and lymphocytic infiltration. Appropriate antibiotics were given accordingly. The diagnosis of $\mathrm{BF}$ was considered. The coronal view of the abdominal CT showed a decrease in pleural effusion and an increase in perihepatic effusion on November 2, 2020. Endoscopic retrograde cholangiopancreatography (ERCP) arranged 3 days later showed contrast extravasation into the right pleural space, suggestive of BF (Figure 5A). We carefully super-selected the distal part of the fistula using a 0.035 guidewire through the scope. Next, a 5 FR guiding catheter was advanced to the ostium of fistula near

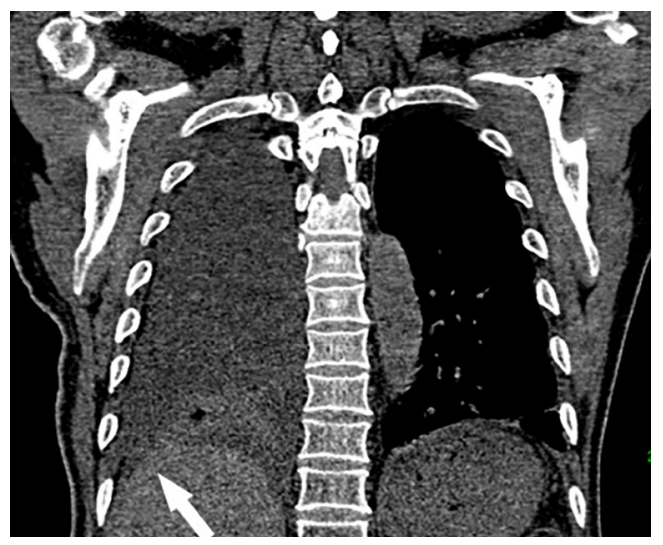

Figure 4 Computed tomography showed right pleural effusion and perihepatic effusion (white arrow).

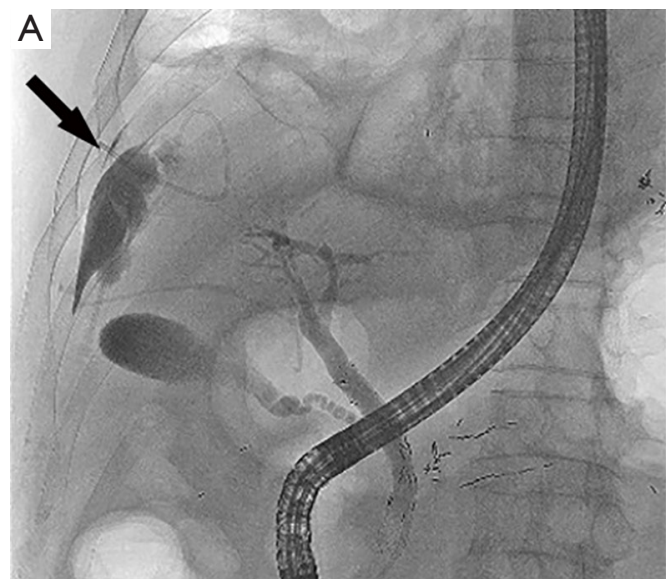

Figure 5 Endoscopic retrograde cholangiopancreatography (ERCP) was performed to embolize the ostium of the fistula. (A) ERCP showed contrast extravasation into the right pleural space, suggestive of biliopleural fistula (BF) (black arrow). (B) Two microcoils (white arrow) and $350-560 \mu \mathrm{m}$ gelatin sponge particles were injected into the main branch of the fistula via the catheter.

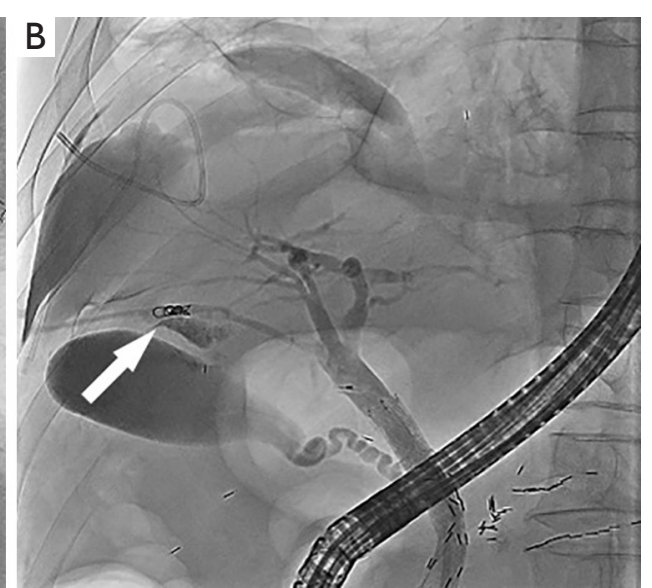

the hepatic edge over the guidewire. We then used a $5-\mathrm{mL}$ syringe to push 2 microcoils (18S-4-2 Tornado, Cook Medical) with $5 \mathrm{~mL}$ of saline solution for embolization. The ostium of the fistula was embolized using absorbable gelatin sponge particles (350-560 $\mu \mathrm{m}$, Hangzhou Alicon Medical Inc., Hangzhou, China), followed by embolization of the 2 microcoils. The injection speed of the gelatin sponge particles was slow, and the regurgitation of embolic materials was avoided as much as possible. After waiting for a few minutes, control cholangiography demonstrated total occlusion of the fistula without any complication (Figure 5B). A 7.0 FR nasobiliary catheter (207225, EndoFlex, Voerde, Germany) was finally inserted into the CBD. The pleural drainage fluid decreased and cleared gradually. During a repeat cholangiography procedure performed 9 days later, the fistula could no longer be visualized (Figure 6). The nasobiliary catheter and pleural drainage tube were immediately removed. Liver function re-examination on November 20, 2020, showed that the TBIL and DBIL levels were 12.2 and $9.1 \mu \mathrm{mol} / \mathrm{L}$, respectively. The CA199 level had decreased to $131 \mathrm{U} / \mathrm{mL}$, and the CEA level had decreased to $6.09 \mathrm{ng} / \mathrm{mL}$. The patient recovered uneventfully and was discharged 45 days after admission. CT follow-up was performed 2 months later, and the right pleural effusion and perihepatic effusion could no longer be visualized (Figure 7).

\section{Discussion}

$\mathrm{BF}$, a fistulous communication between the biliary duct and pleural space, is a very rare clinical entity. The most 


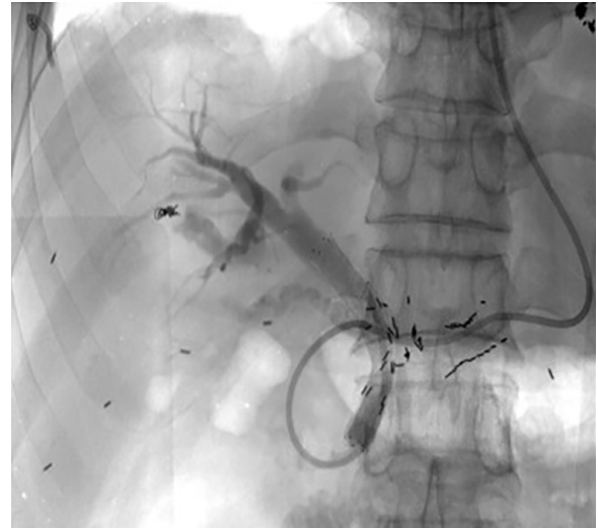

Figure 6 Cholangiography performed 9 days later showed the fistula could no longer be visualized.

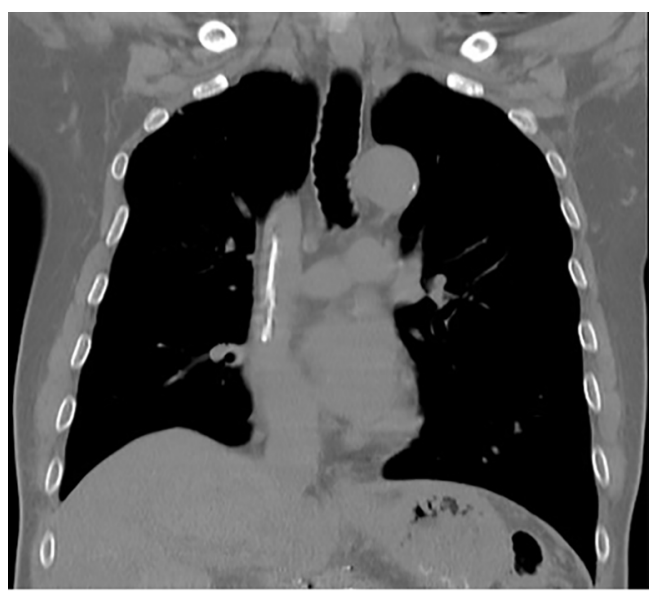

Figure 7 Computed tomography (CT) was performed 2 months later, and right pleural effusion and perihepatic effusion could no longer be visualized.

common cause of BF is iatrogenic injury to the bile ducts (1), and other causes include thoracoabdominal trauma and spontaneity. Early diagnosis of $\mathrm{BF}$ is usually difficult due to the insidious onset. BF may lead to serious outcomes and even death (2). Biliary stent insertion and percutaneous transhepatic cholangiodrainage, which can rapidly release biliary obstruction, has become the preferred palliative treatment for patients with malignant biliary obstruction. However, there is a potential risk of stent occlusion and the need for repeat procedures, and the therapy may not provide benefit in prolonging survival. Studies have reported that irradiation biliary stent has longer patency and better survival compared with conventional metal stent. Furthermore, it does not increase the difficulty of the technique or the incidence of treatment-related complications $(3,4)$. BF secondary to PTCD and irradiation biliary stent insertion is a rare but serious complication, which may arise due to the passage of a catheter through the costophrenic angle before crossing the hepatic parenchyma and into the bile duct. The elevated pressure gradient in the biliary tract can drive the bile leak back into the pleural cavity. We suspect that the following might have caused the accessing of bile duct through the costophrenic angle in this patient: (I) smaller liver volume, (II) higher puncture point, and (III) larger thoracic expansion. It is important to evaluate the positional relationship between the puncture point and costophrenic angle when the patient is breathing in deeply. We think the self-expanding stent was expanded incompletely, and the leaky bile flowed to the lower pressure side (pleural cavity). Hence, it is essential to block the fistula with the combination of a coil and gelatin sponge particle.

The clinical symptoms of BF are dyspnea and chest suffocation. Early diagnosis can reduce mortality. The diagnosis of BF should be considered when a patient with obstructive jaundice after a PTCD catheterization develops a right massive pleural effusion. Ultrasound and CT scans can identify pleural effusion and dilatation of the IHD, but they cannot determine the location of the fistula ostium. Confirmation of BF is obtained when bilious greenish fluid is collected after thoracentesis and when the pleural fluid TBIL to serum TBIL ratio is $>3.0$ (1).

The treatment of $\mathrm{BF}$ includes conservative treatment, interventional or endoscopic treatment, and surgical treatment. However, most patients cannot tolerate surgical intervention. The principles of treatment are relief of the biliary obstruction and closure of the fistula (5). Closure of the fistula is optional or can be used as second-line treatment when the fistula is large or when biliary drainage is not successful in closing the BF. The amount of pleural effusion will decrease rapidly with a drainage tube. Studies have reported that the early institution of effective biliary drainage is key to the success of the treatment (6). In this case, the symptoms of chest suffocation and shortness of breath were improved under the conservative treatment. However, the bile-like substance from the pleural drainage tube was still greenish and copious, and an increase of perihepatic effusion and nondilatation of the IHD were detected in the CT scan. Hence, we super-selected the offending bile duct with a catheter and occluded the fistula with coils and gelatin sponge granules under endoscopy. A nasobiliary catheter was finally inserted. The relief of 
biliary obstruction and closure of the fistula were completed synchronously. The coil was a nonabsorbable embolic material with good operability and could be pushed easily and visualized clearly under $\mathrm{X}$-ray; however, it is difficult to achieve complete occlusion with this coil. A gelatin sponge particle, which is an absorbable embolic material, is a great addition to coil embolization. The combination of the 2 embolic materials can achieve complete embolism and has been widely used in treatment of hemobilia and occlusion of the puncture route after PTCD (7). The injection speed of the gelatin sponge particles should be slow and the regurgitation of embolic materials should be avoided. A 7.0 FR nasobiliary catheter was finally inserted into the CBD to minimize the effect of regurgitation. This mature technique has a low complication rate and significant therapeutic effect. Of course, we hope that this case report will raise awareness of this rare but serious complication and can provide novel approaches for the clinical treatment of BF, which requires cooperation between multiple disciplines.

\section{Acknowledgments}

Funding: This study was supported by the National Natural Science Foundation of China (No. 81971716). The funders of the study had no role in the study design, collection, analysis, or interpretation of data.

\section{Footnote}

Conflicts of Interest: All authors have completed the ICMJE uniform disclosure form (available at https://dx.doi. org/10.21037/qims-21-447). The authors have no conflicts of interest to declare.

Ethical Statement: The authors are accountable for all aspects of the work in ensuring that questions related to the accuracy or integrity of any part of the work are appropriately investigated and resolved. All procedures performed in studies involving human participants were in accordance with the ethical standards of the institutional and/or national research committee(s) and with the Helsinki Declaration (as revised in 2013). Written informed consent was obtained from the patient for publication of this case report and accompanying images. A copy of the written consent is available for review by the editorial office of this journal.

Open Access Statement: This is an Open Access article distributed in accordance with the Creative Commons Attribution-NonCommercial-NoDerivs 4.0 International License (CC BY-NC-ND 4.0), which permits the noncommercial replication and distribution of the article with the strict proviso that no changes or edits are made and the original work is properly cited (including links to both the formal publication through the relevant DOI and the license). See: https://creativecommons.org/licenses/by-nc-nd/4.0/.

\section{References}

1. Nikpour AM, Knebel RJ, Cheng D. Diagnosis and Management of Postoperative Biliary Leaks. Semin Intervent Radiol 2016;33:307-12.

2. Yi-Yung Yu E, Yang FS, Chiu YJ, Tsai FJ, Lu CC, Yang JS. Late onset of biliopleural fistula following percutaneous transhepatic biliary drainage: a case report. Biomedicine (Taipei) 2018;8:6.

3. Zhu HD, Guo JH, Zhu GY, He SC, Fang W, Deng G, Qin YL, Li GZ, Coldwell DM, Teng GJ. A novel biliary stent loaded with (125)I seeds in patients with malignant biliary obstruction: preliminary results versus a conventional biliary stent. J Hepatol 2012;56:1104-11.

4. Zhu HD, Guo JH, Huang M, Ji JS, Xu H, Lu J, et al. Irradiation stents vs. conventional metal stents for unresectable malignant biliary obstruction: A multicenter trial. J Hepatol 2018;68:970-7.

5. LeBedis CA, Bates DDB, Soto JA. Iatrogenic, blunt, and penetrating trauma to the biliary tract. Abdom Radiol (NY) 2017;42:28-45.

6. De Meester X, Vanbeckevoort D, Aerts R, Van Steenbergen W. Biliopleural fistula as a late complication of percutaneous transhepatic cholangioscopy. Endoscopy 2005;37:183.

7. Cao H, Liu J, Li T, Cao G, Xu G, Zhai S, Xue J, Wang Z, Shi S, Bai W. Interventional therapy for the treatment of severe hemobilia after percutaneous transhepatic cholangial drainage: a case series. Int Surg 2013;98:223-8.

Cite this article as: Gao F, Pan T, Wang Y, Zhu G, Feng Y, Zhu C, Guo J. Biliopleural fistula following percutaneous transhepatic cholangiodrainage and irradiation biliary stent insertion successfully treated with coil embolization: a case description. Quant Imaging Med Surg 2022;12(2):1636-1640. doi: 10.21037/qims-21-447 